\title{
Lean buffering in serial production lines with non-exponential machines
}

\author{
Emre Enginarlar ${ }^{1}$, Jingshan $\mathbf{L i}^{2}$, and Semyon M. Meerkov ${ }^{3}$ \\ 1 Decision Applications Division, Los Alamos National Laboratory, \\ Los Alamos, NM 87545, USA \\ 2 Manufacturing Systems Research Laboratory, GM Research and Development Center, \\ Warren, MI 48090-9055, USA \\ 3 Department of Electrical Engineering and Computer Science, University of Michigan, \\ Ann Arbor, MI 48109-2122, USA (e-mail: smm@eecs.umich.edu)
}

\begin{abstract}
In this paper, lean buffering (i.e., the smallest level of buffering necessary and sufficient to ensure the desired production rate of a manufacturing system) is analyzed for the case of serial lines with machines having Weibull, gamma, and log-normal distributions of up- and downtime. The results obtained show that: (1) the lean level of buffering is not very sensitive to the type of up- and downtime distributions and depends mainly on their coefficients of variation, $C V_{u p}$ and $C V_{\text {down }}$; (2) the lean level of buffering is more sensitive to $C V_{\text {down }}$ than to $C V_{u p}$ but the difference in sensitivities is not too large (typically, within 20\%). Based on these observations, an empirical law for calculating the lean level of buffering as a function of machine efficiency, line efficiency, the number of machines in the system, and $C V_{u p}$ and $C V_{\text {down }}$ is introduced. It leads to a reduction of lean buffering by a factor of up to 4 , as compared with that calculated using the exponential assumption. It is conjectured that this empirical law holds for any unimodal distribution of up- and downtime, provided that $C V_{u p}$ and $C V_{\text {down }}$ are less than 1.
\end{abstract}

Keywords: Lean production systems - Serial lines - Non-exponential machine reliability model - Coefficients of variation - Empirical law

\section{Introduction}

\subsection{Goal of the study}

The smallest buffer capacity, which is necessary and sufficient to achieve the desired throughput of a production system, is referred to as lean buffering. In (Enginarlar et al., 2002, 2003a), the problem of lean buffering was analyzed for the case of 
serial production lines with exponential machines, i.e., the machines having upand downtime distributed exponentially. The development was carried out in terms of normalized buffer capacity and production system efficiency. The normalized buffer capacity was introduced as

$k=\frac{N}{T_{\text {down }}}$,

where $N$ denoted the capacity of each buffer and $T_{\text {down }}$ the average downtime of each machine in units of cycle time (i.e., the time necessary to process one part by a machine). Parameter $k$ was referred to as the Level of Buffering $(L B)$. The production line efficiency was quantified as

$E=\frac{P R_{k}}{P R_{\infty}}$

where $P R_{k}$ and $P R_{\infty}$ represented the production rate of the line (i.e., the average number of parts produced by the last machine per cycle time) with $L B$ equal to $k$ and infinity, respectively. The smallest $k$, which ensured the desired $E$, was denoted as $k_{E}$ and referred to as the Lean Level of Buffering $(L L B)$.

Using parameterizations (1) and (2), Enginarlar et al., (2002, 2003a) derived closed formulas for $k_{E}$ as a function of system characteristics. For instance, in the case of two-machines lines, it was shown that (Enginarlar et al., 2002)

$k_{E}^{\exp }= \begin{cases}\frac{2 e(E-e)}{1-E}, & \text { if } e<E, \\ 0, & \text { otherwise. }\end{cases}$

Here the superscript exp indicates that the machines have exponentially distributed up- and downtime, and $e$ denotes machine efficiency in isolation, i.e.,

$e=\frac{T_{u p}}{T_{u p}+T_{\text {down }}}$,

where $T_{u p}$ is the average uptime in units of cycle time. For the case of $M>2$ machine serial lines, the following formula had been derived (Enginarlar et al., 2003a):

$$
k_{E}^{e x p}(M \geq 3)= \begin{cases}\frac{e(1-Q)(e Q+1-e)(e Q+2-2 e)(2-Q)}{Q\left(2 e-2 e Q+e Q^{2}+Q-2\right)} \times \\ \ln \left(\frac{E-e E+e E Q-1+e-2 e Q+e Q^{2}+Q}{(1-e-Q+e Q)(E-1)}\right), & \text { if } e<E^{\frac{1}{M-1}}, \\ 0, & \text { otherwise }\end{cases}
$$

where

$$
\begin{aligned}
Q & =1-E^{\frac{1}{2}\left[1+\left(\frac{M-3}{M-1}\right)^{M / 4}\right]}+\left(E^{\frac{1}{2}\left[1+\left(\frac{M-3}{M-1}\right)^{M / 4}\right]}-E^{\frac{M-2}{M-1}}\right) \\
& \times \exp \left\{-\left(\frac{E^{\frac{1}{M-1}}-e}{1-E}\right)\right\} .
\end{aligned}
$$


This formula is exact for $M=3$ and approximate for $M>3$.

Initial results on lean buffering for non-exponential machines have been reported in (Enginarlar et al., 2002). Two distributions of up- and downtime have been considered (Rayleigh and Erlang). It has been shown that $L L B$ for these cases is smaller than that for the exponential case. However, (Enginarlar et al., 2002) did not provide a sufficiently complete characterization of lean buffering in non-exponential production systems. In particular, it did not quantify how different types of up- and downtime distributions affect $L L B$ and did not investigate relative effects of uptime vs. downtime on $L L B$.

The goal of this paper is to provide a method for selecting $L L B$ in serial lines with non-exponential machines. We consider Weibull, gamma, and log-normal reliability models under various assumptions on their parameters. This allows us to place their coefficients of variations at will and study $L L B$ as a function of up- and downtime variability. Moreover, since each of these distributions is defined by two parameters, selecting them appropriately allows us to analyze the lean buffering for 26 various shapes of density functions, ranging from almost delta-function to almost uniform. This analysis leads to the quantification of both influences of distribution shapes on $L L B$ and effects of up- and downtime on $L L B$. Based of these results, we develop a method for selecting $L L B$ in serial lines with Weibull, gamma, and log-normal reliability characteristics and conjecture that the same method can be used for selecting $L L B$ in serial lines with arbitrary unimodal distributions of upand downtime.

\subsection{Motivation for considering non-exponential machines}

The case of non-exponential machines is important for at least two reasons:

First, in practice the machines often have up- and downtime distributed nonexponentially. As the empirical evidence (Inman, 1999) indicates, the coefficients of variation, $C V_{u p}$ and $C V_{\text {down }}$ of these random variables are often less than 1; thus, the distributions cannot be exponential. Therefore, an analytical characterization of $k_{E}$ for non-exponential machines is of theoretical importance.

Second, such a characterization is of practical importance as well. Indeed, it can be expected that $k_{E}^{e x p}$ is the upper bound of $k_{E}$ for $C V<1$ and, moreover, $k_{E}$ might be substantially smaller than $k_{E}^{e x p}$. This implies that a smaller buffer capacity is necessary to achieve the desired line efficiency $E$ when the machines are nonexponential. Thus, selecting $L L B$ based on realistic, non-exponential reliability characteristics would lead to increased leanness of production systems.

\subsection{Difficulties in studying the non-exponential case}

Analysis of lean buffering in serial production lines with non-exponential machines is complicated, as compared with the exponential case, by the reasons outlined in Table 1 . Especially damaging is the first one, which practically precludes analytical investigation. The other reasons lead to a combinatorially increasing number of cases to be investigated. In this work, we partially overcome these difficulties by 
Table 1. Difficulties of the non-exponential case as compared with the exponential one

\begin{tabular}{ll}
\hline Exponential case & Non-exponential case \\
\hline $\begin{array}{l}\text { Analytical methods for evaluating } \\
P R \text { are available }\end{array}$ & $\begin{array}{l}\text { No analytical methods for evaluating } \\
P R \text { are available }\end{array}$ \\
$\begin{array}{l}\text { Machine up- and downtimes are distributed } \\
\text { identically (i.e., exponentially). }\end{array}$ & $\begin{array}{l}\text { Machine up- and downtimes may } \\
\text { have different distributions. }\end{array}$ \\
$\begin{array}{l}\text { Coefficients of variation of machine } \\
\text { up- and downtimes are identical } \\
\text { and equal to 1. }\end{array}$ & $\begin{array}{l}\text { Coefficients of variation of machine } \\
\text { up- and downtimes may take arbitrary } \\
\text { positive values and may be } \\
\text { non-identical. }\end{array}$ \\
$\begin{array}{l}\text { All machines in the system have the } \\
\text { same type of up- and downtime distributions } \\
\text { (i.e., exponential). }\end{array}$ & $\begin{array}{l}\text { Each machine in the system may have } \\
\text { different types of up- and downtime }\end{array}$ \\
\hline
\end{tabular}

using numerical simulations and by restricting the number of distributions and coefficients of variation analyzed.

\subsection{Related literature}

The majority of quantitative results on buffer capacity allocation in serial production lines address the case of exponential or geometric machines (Buzacott, 1967; Caramanis, 1987; Conway et al., 1988; Smith and Daskalaki, 1988; Jafari and Shanthikumar, 1989; Park, 1993; Seong et al., 1995; Gershwin and Schor, 2000). Just a few numerical/empirical studies are devoted to the non-exponential case. Specifically, two-stage coaxian type completion time distributions are considered by Altiok and Stidham (1983), Chow (1987), Hillier and So (1991a,b), and the effects of log-normal processing times are analyzed by Powell (1994), Powell and Pyke (1998), Harris and Powell (1999). These papers consider lines with reliable machines having random processing time. Another approach is to develop methods to extend the results obtained for such cases to unreliable machines with deterministic processing time (Tempelmeier, 2003). Phase-type distributions to model random processing time and reliability characteristics are analyzed by Altiok $(1985,1989)$, Altiok and Ranjan (1989), Yamashita and Altiok (1998), but the resulting methods are computationally intensive and can be used only for short lines with small buffers (e.g., two-machine lines with buffers of capacity less than six). Finally, as it was mentioned in the Introduction, initial results on lean level of buffering in serial lines with Rayleigh and Erlang machines have been reported in (Enginarlar et al., 2002). 


\subsection{Contributions of this paper}

The main results derived in this paper are as follows:

- $L L B$ is not very sensitive to the type of up- and downtime distributions and depends mostly on their coefficients of variation $\left(C V_{u p}\right.$ and $\left.C V_{d o w n}\right)$.

- $L L B$ is more sensitive to $C V_{\text {down }}$ than to $C V_{u p}$, but this difference in sensitivities is not too large (typically, within $20 \%$ ).

- In serial lines with $M$ machines having Weibull, gamma, and log-normal distributions of up- and downtime with $C V_{u p}$ and $C V_{\text {down }}$ less than $1, L L B$ can be selected using the following upper bound:

$$
\begin{aligned}
& k_{E}\left(M, E, e, C V_{u p}, C V_{\text {down }}\right) \\
& \leq \frac{\max \left\{0.25, C V_{u p}\right\}+\max \left\{0.25, C V_{\text {down }}\right\}}{2} k_{E}^{\exp }(M, E, e),
\end{aligned}
$$

where $k_{E}^{e x p}$ is given by (5), (6). This bound is referred to as the empirical law. It is conjectured that this bound holds for all unimodal up- and downtime distributions with $C V_{u p}<1$ and $C V_{\text {down }}<1$.

- Although for some values of $C V_{u p}$ and $C V_{d o w n}$, bound (7) may not be too tight, it still leads to a reduction of lean buffering by a factor of up to 4, as compared to $L L B$ based on the exponential assumption.

\subsection{Paper organization}

In Section 2, the model of the production system under consideration is introduced and the problems addressed are formulated. Section 3 describes the approach of this study. Sections 4 and 5 present the main results pertaining, respectively, to systems with machines having identical and non-identical coefficients of variation of up- and downtime. In Section 6, serial lines with machines having arbitrary, i.e., general, reliability models are discussed. Finally, in Section 7, the conclusions are formulated.

\section{Model and problem formulation}

\subsection{Model}

The block diagram of the production system considered in this work is shown in Figure 1, where the circles represent the machines and the rectangles are the buffers. Assumptions on the machines and buffers, described below, are similar to those of (Enginarlar et al., 2003a) with the only difference that up- and downtime distributions are not exponential. Specifically, these assumptions are:

(i) Each machine $m_{i}, i=1, \ldots, M$, has two states: up and down. When up, the machine is capable of processing one part per cycle time; when down, no production takes place. The cycle times of all machines are the same. 


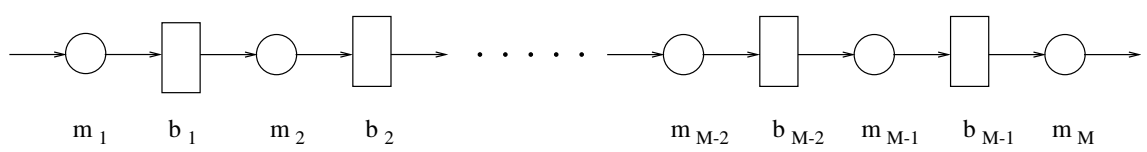

Fig. 1. Serial production line

(ii) The up- and downtime of each machine are random variables measured in units of the cycle time. In other words, uptime (respectively, downtime) of length $t \geq 0$ implies that the machine is up (respectively, down) during $t$ cycle times. The upand downtime are distributed according to one of the following probability density functions, referred to as reliability models:

(a) Weibull, i.e.,

$$
\begin{aligned}
f_{u p}^{W}(t) & =p^{P} e^{-(p t)^{P}} P t^{P-1}, \\
f_{\text {down }}^{W}(t) & =r^{R} e^{-(r t)^{R}} R t^{R-1},
\end{aligned}
$$

where $f_{u p}^{W}(t)$ and $f_{\text {down }}^{W}(t)$ are the probability density functions of up- and downtime, respectively and $(p, P)$ and $(r, R)$ are their parameters. (Here, and in the subsequent distributions, the parameters are positive real numbers). These distributions are denoted as $W(p, P)$ and $W(r, R)$, respectively.

(b) Gamma, i.e.,

$$
\begin{aligned}
f_{u p}^{g}(t) & =p e^{-p t} \frac{(p t)^{P-1}}{\Gamma(P)}, \\
f_{\text {down }}^{g}(t) & =r e^{-r t} \frac{(r t)^{R-1}}{\Gamma(R)},
\end{aligned}
$$

where $\Gamma(x)$ is the gamma function, $\Gamma(x)=\int_{0}^{\infty} s^{x-1} e^{-s} d s$. These distributions are denoted as $g(p, P)$ and $g(r, R)$, respectively.

(c) Log-normal, i.e.,

$$
\begin{aligned}
f_{\text {up }}^{L N}(t) & =\frac{1}{\sqrt{2 \pi} P t} e^{-\frac{(\ln (t)-p)^{2}}{2 P^{2}}}, \\
f_{\text {down }}^{L N}(t) & =\frac{1}{\sqrt{2 \pi} R t} e^{-\frac{(\ln (t)-r)^{2}}{2 R^{2}}} .
\end{aligned}
$$

We denote these distributions as $L N(p, P)$ and $L N(r, R)$, respectively.

The expected values, variances, and coefficients of variation of distributions (8)-(10) are given in Table 2.

(iii) The parameters of distributions (8)-(10) are selected so that the machine efficiencies, i.e.,

$e=\frac{T_{u p}}{T_{u p}+T_{\text {down }}}$,

and, moreover, $T_{u p}, T_{\text {down }}, C V_{u p}$, and $C V_{\text {down }}$ of all machines are identical for all reliability models, i.e.,

$$
T_{u p}=p^{-1} \Gamma\left(1+\frac{1}{P}\right) \quad \text { (Weibull) }
$$


Table 2. Expected value, variance, and coefficient of variation of up- and downtime distributions considered

\begin{tabular}{cllc}
\hline & Gamma & Weibull & Log-normal \\
\hline$T_{\text {up }}$ & $P / p$ & $p^{-1} \Gamma(1+1 / P)$ & $e^{p+P^{2} / 2}$ \\
$T_{\text {down }}$ & $R / r$ & $r^{-1} \Gamma(1+1 / R)$ & $e^{r+R^{2} / 2}$ \\
$\sigma_{\text {up }}^{2}$ & $P / p^{2}$ & $p^{-2}\left[\Gamma(1+2 / P)-\Gamma^{2}(1+1 / P)\right]$ & $e^{2 p+P^{2}\left(e^{P^{2}}-1\right)}$ \\
$\sigma_{\text {down }}^{2}$ & $R / r^{2}$ & $r^{-2}\left[\Gamma(1+2 / R)-\Gamma^{2}(1+1 / R)\right]$ & $e^{2 r+R^{2}\left(e^{R^{2}}-1\right)}$ \\
$C V_{\text {up }}$ & $1 / \sqrt{P}$ & $\sqrt{\Gamma(1+2 / P)-\Gamma^{2}(1+1 / P)} / \Gamma(1+1 / P)$ & $\sqrt{e^{P^{2}}-1}$ \\
$C V_{\text {down }}$ & $1 / \sqrt{R}$ & $\sqrt{\Gamma(1+2 / R)-\Gamma^{2}(1+1 / R)} / \Gamma(1+1 / R)$ & $\sqrt{e^{R^{2}}-1}$ \\
\hline
\end{tabular}

$$
\begin{aligned}
& =\frac{P}{p} \quad \text { (gamma) } \\
& =e^{p+P^{2} / 2} \quad \text { (log-normal); } \\
& T_{\text {down }}=r^{-1} \Gamma(1+1 / R) \quad(\text { Weibull }) \\
& =\frac{R}{r} \quad \text { (gamma) } \\
& =e^{r+R^{2} / 2} \quad \text { (log-normal); } \\
& C V_{u p}=\frac{\sqrt{\Gamma(1+2 / P)-\Gamma^{2}(1+1 / P)}}{\Gamma(1+1 / P)} \quad \text { (Weibull) } \\
& =\frac{1}{\sqrt{P}} \quad \text { (gamma) } \\
& =\sqrt{e^{P^{2}}-1} \quad \text { (log-normal); } \\
& C V_{\text {down }}=\frac{\sqrt{\Gamma(1+2 / R)-\Gamma^{2}(1+1 / R)}}{\Gamma(1+1 / R)} \quad \text { (Weibull) } \\
& =\frac{1}{\sqrt{R}} \quad \text { (gamma) } \\
& =\sqrt{e^{R^{2}}-1} \quad \text { (log-normal). }
\end{aligned}
$$

(iv) Buffer $b_{i}, i=1, \ldots, M-1$ is of capacity $0 \leq N \leq \infty$.

(v) Machine $m_{i}, i=2, \ldots, M$, is starved at time $t$ if it is up at time $t$, buffer $b_{i-1}$ is empty at time $t$ and $m_{i-1}$ does not place any work in this buffer at time $t$. Machine $m_{1}$ cannot be starved.

(vi) Machine $m_{i}, i=1, \ldots, M-1$, is blocked at time $t$ if it is up at time $t$, buffer $b_{i}$ is full at time $t$ and $m_{i+1}$ fails to take any work from this buffer at time $t$. Machine $m_{M}$ cannot be blocked. 


\section{Remark 1 .}

- Assumptions (i)-(iii) imply that all machines are identical from all points of view except, perhaps, for the nature of up- and downtime distributions. The buffers are also assumed to be of equal capacity (see (iv)). We make these assumptions in order to provide a compact characterization of lean buffering.

- Assumption (ii) implies, in particular, that time-dependent, rather than operation-dependent failures, are considered. This failure mode simplifies the analysis and results in just a small difference in comparison with operationdependent failures.

\subsection{Notations}

Each machine considered in this paper is denoted by a pair

$\left[D_{\text {up }}(p, P), D_{\text {down }}(r, R)\right]_{i}, \quad i=1, \ldots, M$,

where $D_{u p}(p, P)$ and $D_{\text {down }}(r, R)$ represent, respectively, the distributions of upand downtime of the $i$-th machine in the system, $D_{u p}$ and $D_{\text {down }} \in\{W, g, L N\}$. The serial line with $M$ machines is denoted as

$\left\{\left[D_{\text {up }}, D_{\text {down }}\right]_{1}, \ldots,\left[D_{\text {up }}, D_{\text {down }}\right]_{M}\right\}$.

If all machines have identical distribution of uptimes and downtimes, the line is denoted as

$\left\{\left[D_{\text {up }}(p, P), D_{\text {down }}(r, R)\right]_{i}, i=1, \ldots, M\right\}$.

If, in addition, the types of up- and downtime distributions are the same, the notation for the line is

$\left\{[D(p, P), D(r, R)]_{i}, i=1, \ldots, M\right\}$.

Finally, if up- and downtime distributions of the machines are not necessarily $W$, $g$, or $L N$ but are general in nature, however, unimodal, the line is denoted as

$\left\{\left[G_{\text {up }}, G_{\text {down }}\right]_{1}, \ldots,\left[G_{\text {up }}, G_{\text {down }}\right]_{M}\right\}$.

\subsection{Problems addressed}

Using the parameterizations (1), (2), the model (i)-(vi), and the notations (12)-(16), this paper is intended to

- develop a method for calculating Lean Level of Buffering in production lines (13)-(15) under the assumption that the coefficients of variation of up- and downtime, $C V_{u p}$ and $C V_{\text {down }}$, are identical, i.e., $C V_{u p}=C V_{\text {down }}=C V$;

- develop a method of calculating $L L B$ in production lines (13)-(15) for the case of $C V_{u p} \neq C V_{\text {down }}$;

- extend the results obtained to production lines (16).

Solutions of these problems are presented in Sections 4-6 while Section 3 describes the approach used in this work. 


\section{Approach}

\subsection{General considerations}

Since $L L B$ depends on line efficiency $E$, the calculation of $k_{E}$ requires the knowledge of the production rate, $P R$, of the system. Unfortunately, as it was mentioned earlier, no analytical methods exist for evaluating $P R$ in serial lines with either Weibull, or gamma, or log-normal reliability characteristics. Approximation methods are also hardly applicable since, in our experiences, even 1\%-2\% errors in the production rate evaluation (due to the approximate nature of the techniques) often lead to much larger errors (up to 20\%) in lean buffering characterization. Therefore, the only method available is the Monte Carlo approach based on numerical simulations. To implement this approach, a MATLAB code was constructed, which simulated the operation of the production line defined by assumptions (i)-(vi) of Section 2. Then, a set of representative distributions of up- and downtime was selected and, finally, for each member of this set, $P R$ and $L L B$ were evaluated with guaranteed statistical characteristics. Each of these steps is described below in more detail.

\subsection{Up-and downtime distributions analyzed}

The set of 26 downtime distributions analyzed in this work is shown in Table 3, where the notations introduced in Section 2.1 are used. These distributions are classified according to their coefficients of variation, $C V_{\text {down }}$, which take values from the set $\{0.1,0.25,0.5,0.75,1.0\}$. The analysis of $L L B$ for this set is intended to reveal the behavior of $k_{E}$ as a function of $C V_{\text {down }}$.

To investigate the effect of the average downtime, the distributions of Table 3 have been classified according to $T_{\text {down }}$, which takes values 20 and 100 .

An illustration of a few of the downtime distributions included in Table 3 is given in Figure 2 for $C V_{\text {down }}=0.5$. As one can see, the shapes of the distributions included in Table 3 range from "almost" uniform to "almost" $\delta$-function.

Table 3. Downtime distributions considered

\begin{tabular}{ccc}
\hline$C V_{\text {down }}$ & $T_{\text {down }}=20$ & $T_{\text {down }}=100$ \\
\hline \multirow{2}{*}{0.1} & $g(5,100)$, & $g(1,100)$, \\
& $W(0.048,12.15), L N(2.99,0.1)$ & $W(0.01,12.15), L N(4.602,0.1)$ \\
0.25 & $g(0.8,16)$, & $g(0.16,16)$, \\
& $W(0.046, \quad 4.54), L N(2.97,0.25)$ & $W(0.009, \quad 4.54), L N(4.57,0.25)$ \\
0.5 & $g(0.2,4)$, & $g(0.04,4)$, \\
& $W(0.044,2.1), L N(2.88,0.49)$ & $W(0.009,2.1), L N(4.49,0.49)$ \\
0.75 & $g(0.09,1.8)$, & $g(0.018,1.8)$, \\
& $W(0.046,1.35), L N(2.77,0.66)$ & $W(0.009,1.35), L N(4.38,0.66)$ \\
1.00 & $L N(2.65,0.83)$ & $L N(4.26,0.83)$ \\
\hline
\end{tabular}






Fig. 2. Different distributions with identical coefficients of variation $\left(C V_{\text {down }}=0.5\right)$

The uptime distributions, corresponding to the downtime distributions of Table 3, have been selected as follows: For a given machine efficiency, $e$, the average uptime was chosen as

$T_{u p}=\frac{e}{1-e} T_{\text {down }}$.

Next, $C V_{u p}$ was selected as $C V_{u p}=C V_{\text {down }}$, when the case of identical coefficients of variation of up- and downtime was considered; otherwise $C V_{u p}$ was selected as a constant independent of $C V_{\text {down }}$. Finally, using these $T_{u p}$ and $C V_{u p}$, the distribution of uptime was selected to be the same as that of the downtime, if the case of identical distributions was analyzed; otherwise it was selected as any other distribution from the set $\{W, g, L N\}$. For instance, if the downtime was distributed according to $D_{\text {down }}(r, R)=g(0.018,1.8)$ and $e$ was 0.9 , the uptime distribution was selected as

$D_{u p}(p, P)= \begin{cases}g(0.002,1.8) & \text { for } \quad C V_{u p}=C V_{\text {down }}, \\ g(0.0044,4) & \text { for } \quad C V_{u p}=0.5\end{cases}$

or

$D_{u p}(p, P)=\left\{\begin{array}{lll}L N(6.69,0.47) & \text { for } & C V_{u p}=C V_{d o w n}, \\ L N(2.88,0.49) & \text { for } \quad C V_{u p}=0.5 .\end{array}\right.$

Remark 2. Both $C V_{u p}$ and $C V_{\text {down }}$ considered are less than 1 because, according to the empirical evidence of (Inman, 1999), the equipment on the factory floor often satisfies this condition. In addition, it has been shown by Li and Meerkov (2005) that $C V_{u p}$ and $C V_{\text {down }}$ are less than 1 if the breakdown and repair rates of the machines are increasing functions of time, which often takes place in reality. 


\subsection{Parameters selected}

In all systems analyzed, particular values of $M, E$, and $e$ have been selected as follows:

(a) The number of machines in the system, $M$ : Since, as it was shown in (Enginarlar et al., 2002), $k_{E}^{e x p}$ is not very sensitive to $M$ if $M \geq 10$, the number of machines in the system was selected to be 10 . For verification purposes, we analyzed also serial lines with $M=5$.

(b) Line efficiency, $E$ : In practice, production lines are often operated close to their maximum capacity. Therefore, for the purposes of simulation, $E$ was selected to belong to the set $\{0.85,0.9,0.95\}$. For the purposes of verification, additional values of $E$ analyzed were $\{0.7,0.8\}$.

(c) Machine efficiency, $e$ : Although in practice $e$ may have widely different values (e.g., smaller in machining operations and much larger in assembly), to obtain a manageable set of systems for simulation, $e$ was selected from the set $\{0.85,0.9,0.95\}$. For verification purposes, we considered $e \in\{0.6,0.7,0.8\}$.

\subsection{Systems analyzed}

Specific systems of the form (15) considered in this work are:

$$
\begin{array}{r}
\left\{[W(p, P), W(r, R)]_{i}, i=1, \ldots, 10\right\}, \\
\left\{[g(p, P), g(r, R)]_{i}, i=1, \ldots, 10\right\}, \\
\left\{[L N(p, P), L N(r, R)]_{i}, i=1, \ldots, 10\right\} .
\end{array}
$$

Systems of the form (13) have been formed as follows: For each machine $m_{i}, i=1, \ldots, 10$, the up- and downtime distributions were chosen from the set $\{W, g, L N\}$ equiprobably and independently of each other and all other machines in the system. As a result, the following two lines were selected:

Line 1: $\{(g, W),(L N, L N),(W, g),(g, L N),(g, W)$,

$$
(L N, g),(W, W),(g, g),(L N, W),(g, L N)\}
$$

Line 2: $\{(W, L N),(g, W),(L N, W),(W, g),(g, L N)$,

$$
(g, W),(W, W),(L N, g),(g, W),(L N, L N)\} .
$$

We will use notations $A \in\{(17)\}, A \in\{(18)\}$ or $A \in\{(17),(18)\}$ to indicate that line $A$ is one of (17), or one of (18), and one of (17) and (18), respectively.

Lines (17) and (18) are analyzed in Sections 4 and 5 for the cases of $C V_{u p}=$ $C V_{\text {down }}$ and $C V_{\text {up }} \neq C V_{\text {down }}$, respectively.

\subsection{Evaluation of the production rate}

To evaluate the production rate in systems (17) and (18), using the MATLAB code and the up- and downtime distributions discussed in Sections 3.1-3.3, zero initial 
conditions of all buffers have been assumed and the states of all machines at the initial time moment have been selected "up". The first 100,000 cycle times were considered as warm-up period. The subsequent 1,000,000 cycle times were used for statistical evaluation of $P R$. Each simulation was repeated 10 times, which resulted in $95 \%$ confidence intervals of less than 0.0005 .

\subsection{Evaluation of $L L B$}

The lean buffering, $k_{E}$, necessary and sufficient to ensure line efficiency $E$, was evaluated using the following procedure:

For each model of serial line (13)-(15), the production rate was evaluated first for $N=0$, then for $N=1$, and so on, until the production rate $P R=E \cdot P R_{\infty}$ was achieved. Then $k_{E}$ was determined by dividing the resulting $N_{E}$ by the machine average downtime (in units of the cycle time).

Remark 3. Although, as it is well known (Hillier and So, 1991b), the optimal allocation of a fixed total buffer capacity is non-uniform, to simplify the analysis we consider only uniform allocations. Since the optimal (i.e., inverted bowl) allocation typically results in just $1-2 \%$ throughput improvement in comparison with the uniform allocation, for the sake of simplicity we consider only the latter case.

\section{$4 L L B$ in serial lines with $C V_{u p}=C V_{d o w n}=C V$}

4.1 System $\left\{[D(p, P), D(r, R)]_{i}, i=1, \ldots, 10\right\}$

Figures 3 and 5 present the simulation results for production lines (17) for all distributions of Table 3. These figures are arranged as matrices where the rows and columns correspond to $e \in\{0.85,0.9,0.95\}$ and $E \in\{0.85,0.9,0.95\}$, respectively. Since, due to space considerations, the graphs in Figures 3 and 5 are congested and may be difficult to read, one of them is shown in Figure 4 in a larger scale. (The dashed lines in Figs. 3-5 will be discussed in Sect. 4.3.) Examining these data, the following may be concluded:

- As expected, $k_{E}$ for non-exponential machines is smaller than $k_{E}^{e x p}$. Moreover, $k_{E}$ is a monotonically increasing function of $C V$. In addition, $k_{E}(C V)$ is convex, which implies that reducing larger $C V$ 's leads to larger reduction of $k_{E}$ than reducing smaller $C V$ 's.

- Function $k_{E}(C V)$ seems to be polynomial in nature. In fact, each curve of Figures 3 and 5 can be approximated by a polynomial of an appropriate order. However, since these approximations are "parameter-dependent" (i.e., different polynomials must be used for different $e$ and $E$ ), they are of small practical importance, and, therefore, are not reported here.

- Since for every pair $(E, e)$, corresponding curves of Figures 3 and 5 are identical, it is concluded that $k_{E}$ is not dependent of $T_{u p}$ and $T_{\text {down }}$ explicitly but only through the ratio $e$. In other words, the situation here is the same as in lines with exponential machines (see (5), (6)). 


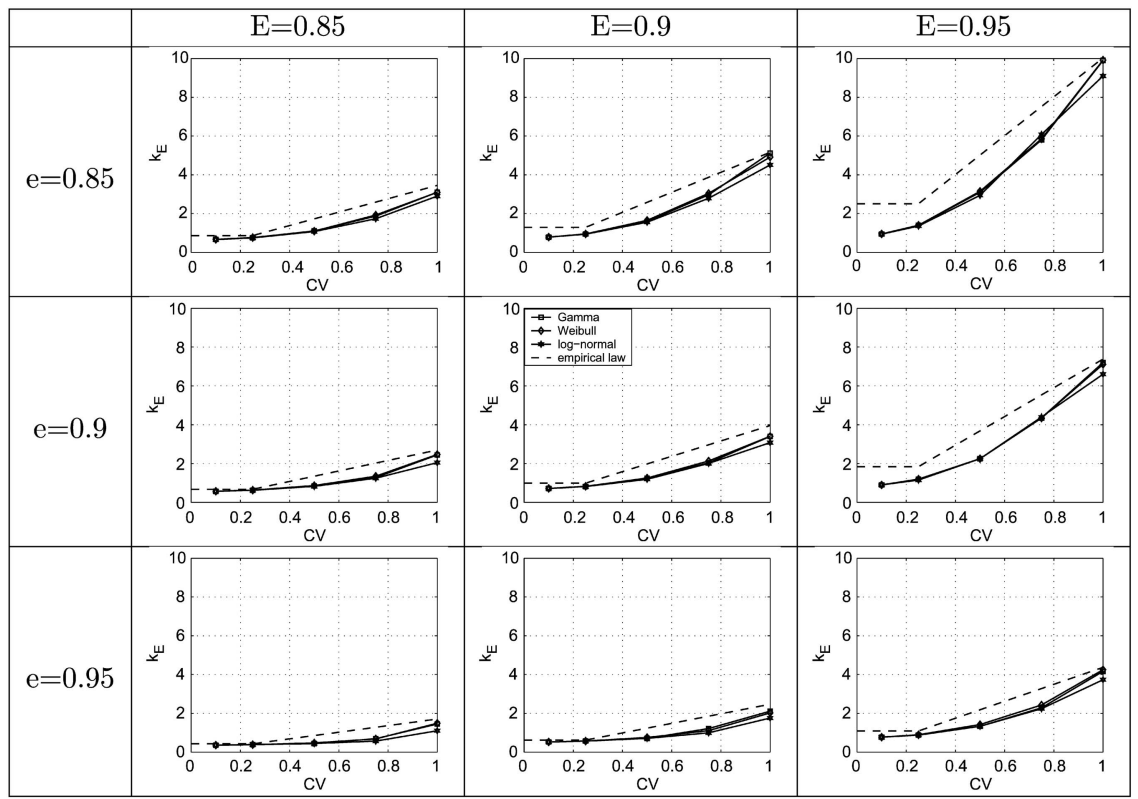

Fig. 3. $L L B$ versus $C V$ for systems (17) with $T_{\text {down }}=20$

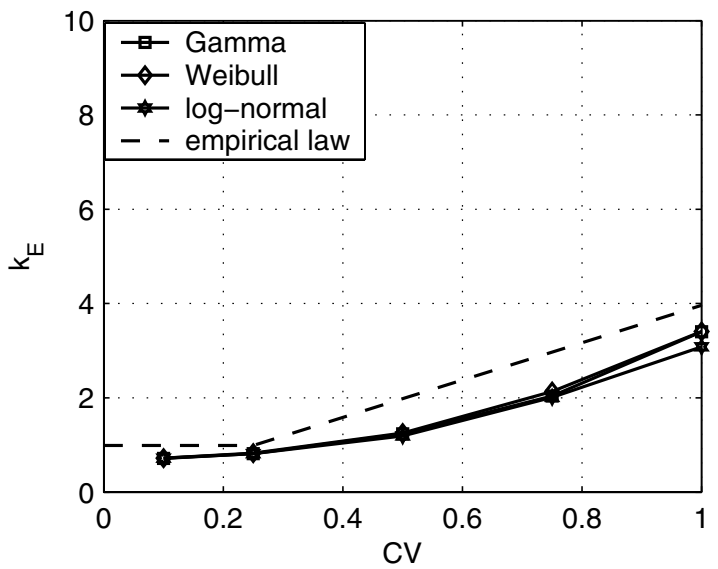

Fig. 4. $L L B$ versus $C V$ for system $\left\{(D(p, P), D(r, R))_{i}, i=1, \ldots, 10\right\}$ with $T_{\text {down }}=$ $20, e=0.9, E=0.9$

- Finally, and perhaps most importantly, the behavior of $k_{E}$ as a function of $C V$ is almost independent of the type of up- and downtime distributions considered. Indeed, let $k_{E}^{A}(C V)$ denote $L L B$ for line $A \in\{(17)\}$ with $C V \in\{0.1,0.25,0.5,0.75,1.0\}$. Then the sensitivity of $k_{E}$ to up- and downtime distributions may be characterized by

$$
\epsilon_{1}(C V)=\max _{A, B \in\{(17)\}}\left|\frac{k_{E}^{A}(C V)-k_{E}^{B}(C V)}{k_{E}^{A}(C V)}\right| \cdot 100 \% .
$$




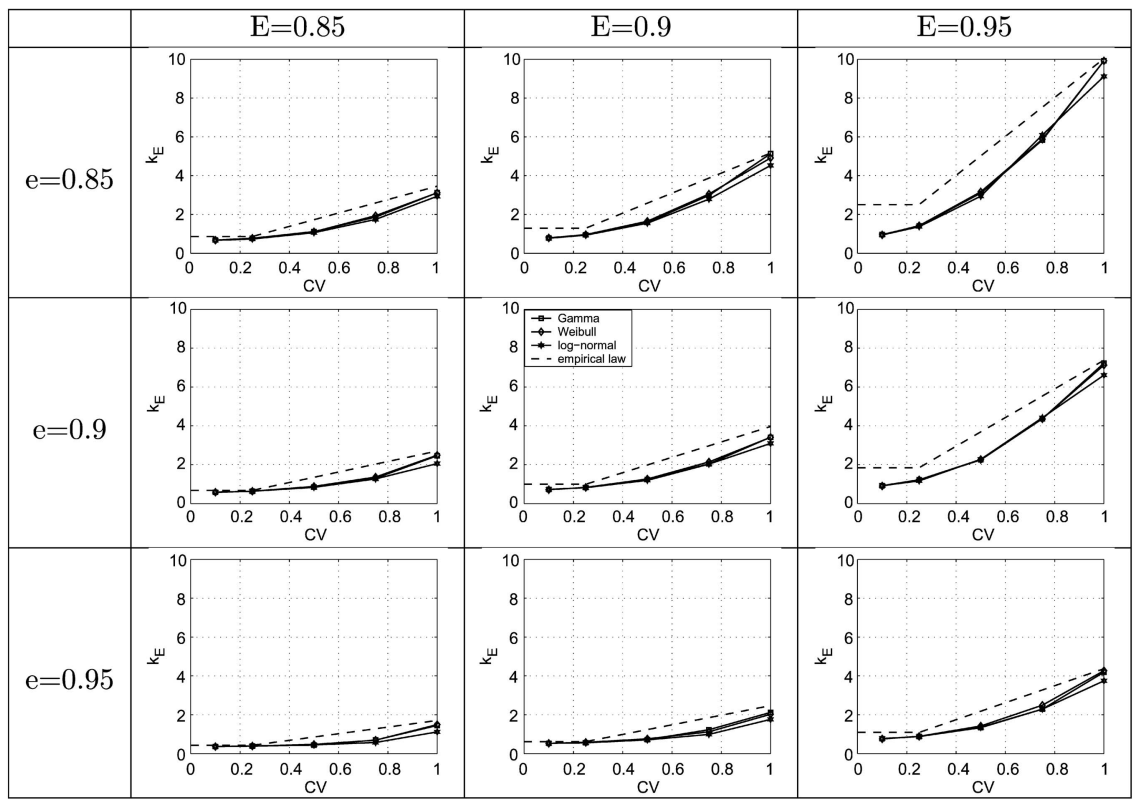

Fig. 5. $L L B$ versus $C V$ for systems (17) with $T_{\text {down }}=100$



Fig. 6. Sensitivity of LLB to the nature of up- and downtime distributions for systems (17) 
Function $\epsilon_{1}(C V)$ is illustrated in Figure 6. As one can see, in most cases it takes values within $10 \%$. Thus, it is possible to conclude that for all practical purposes $k_{E}$ depends on the coefficients of variation of up- and downtime, rather than on actual distribution of these random variables.

\subsection{System $\left\{[D(p, P), D(r, R)]_{1}, \ldots,[D(p, P), D(r, R)]_{10}\right\}$}

Figures 7 and 8 present the simulation results for lines (18), while Figure 9 characterizes the sensitivity of $k_{E}$ to up- and downtime distributions. This sensitivity is calculated according to (19) with the only difference that the max is taken over $A, B \in\{(18)\}$. Based on these data, we affirm that the conclusions formulated in Section 4.1 hold for production lines of the type (13) as well.

\subsection{Empirical law}

\subsubsection{Analytical expression}

Simulation results reported above provide a characterization of $k_{E}$ for $M=10$ and $E$ and $e \in\{0.85,0.9,0.95\}$. How can $k_{E}$ be determined for other values of $M, E$, and $e$ ? Obviously, simulations for all values of these variables are impossible. Even for particular values of $M, E$, and $e$, simulations take a very long time: Figures 3 and 5 required approximately one week of calculations using 25 Sun workstations working in parallel. Therefore, an analytical method for evaluating $k_{E}$ for all values of $M, E, e$, and $C V$ is desirable. Although an exact characterization of the function $k_{E}=k_{E}(M, E, e, C V)$ is all but impossible, results of Sections 4.1 and 4.2 provide an opportunity for introducing an upper bound of $k_{E}$ as a function of all four variables. This upper bound is based on the expression of $k_{E}^{e x p}=k_{E}^{e x p}(M, E, e)$, given by (5), (6), and the fact that all curves of Figures 3, 5 and 7, 8 are below the linear function of $C V$ with the slope $k_{E}^{e x p}$, if $0.25<C V \leq 1$. For $0<C V \leq 0.25$, all curves are below the constant $0.25 k_{E}^{\text {exp }}$. Thus, the following piece-wise linear upper bound for $k_{E}$ may be introduced:

$k_{E}(M, E, e, C V) \leq \max \{0.25, C V\} k_{E}^{e x p}(M, E, e), \quad C V \leq 1$.

This expression, referred to as the empirical law, is illustrated in Figures 3-5 and 7,8 by the broken lines.

The tightness of this bound can be characterized by the function

$\epsilon_{2}(C V)=\max _{A \in\{(17),(18)\}} \frac{k_{E}^{\text {upper bound }}-k_{E}^{A}}{k_{E}^{A}} \cdot 100 \%, \quad C V \leq 1$,

where $k_{E}^{\text {upper bound }}$ is the right-hand-side of (20). Function $\epsilon_{2}(C V)$ is illustrated in Figure 10. Although, as one can see, the empirical law is quite conservative, its usage still leads to up to $400 \%$ reduction of buffering, as compared with that based on the exponential assumption (see Figs. 3, 5 and 7,8).

Remark 4. As it was pointed out above, the curves of Figures 3, 5 and 7, 8 are polynomial in nature. This, along with the quadratic dependence of performance 




Fig. 7. $L L B$ versus $C V$ for systems (18) with $T_{\text {down }}=20$

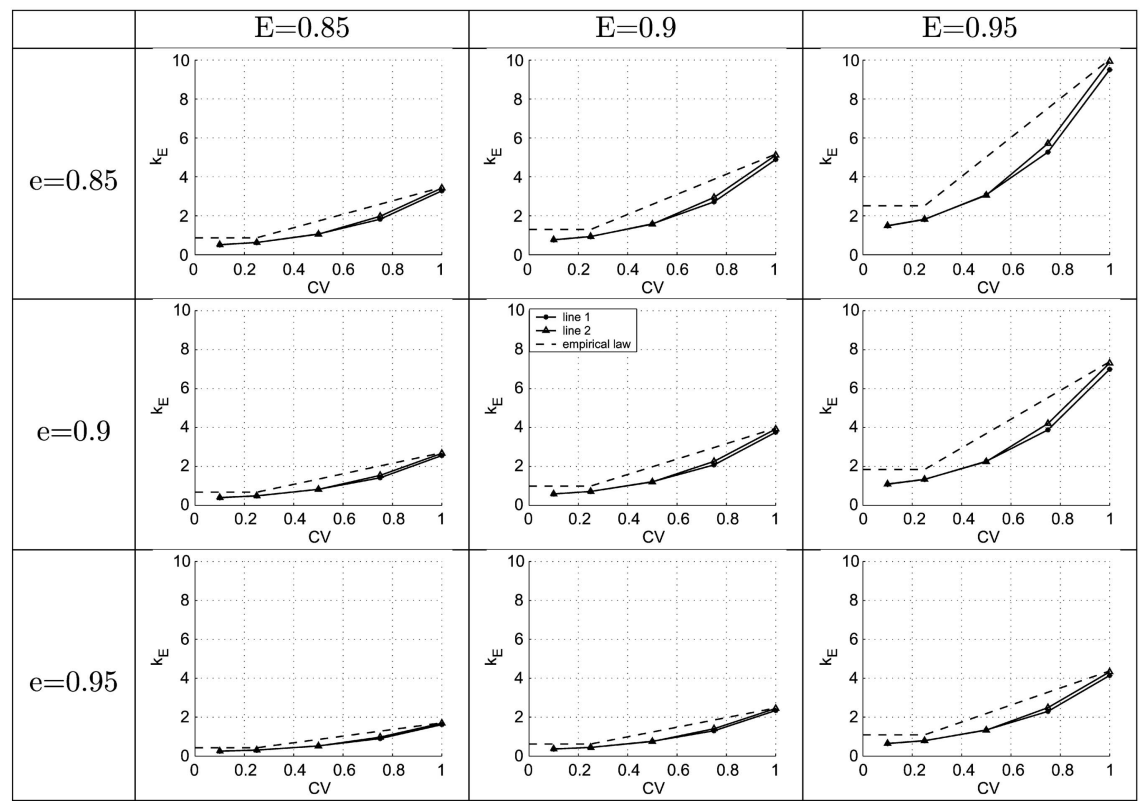

Fig. 8. $L L B$ versus $C V$ for systems (18) with $T_{\text {down }}=100$ 


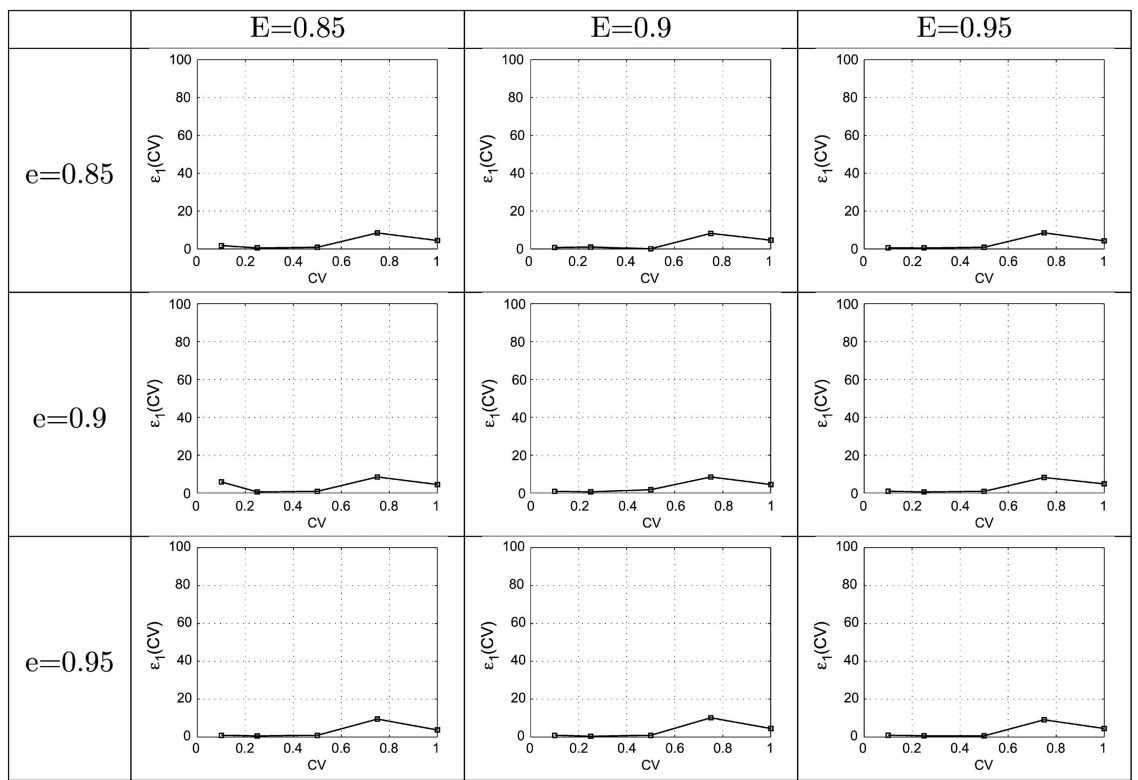

Fig. 9. Sensitivity of $L L B$ to the nature of up- and downtime distributions for systems (18)



Fig. 10. The tightness of the empirical law (20) 


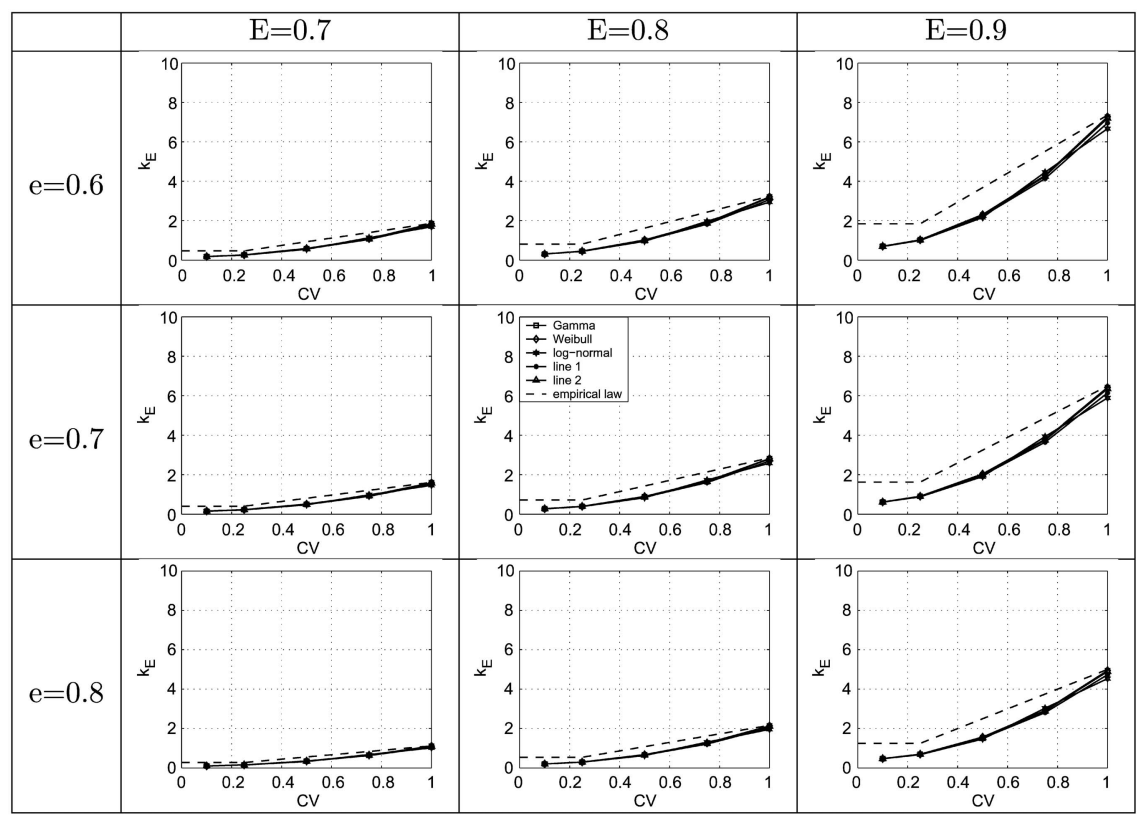

Fig. 11. Verification: $L L B$ versus $C V$ for system $\left\{(D(p, P), D(r, R))_{i}, i=1, \ldots, 5\right\}$ with $T_{\text {down }}=10$

measures on $C V$ in $G / G / 1$ queues, might lead to a temptation to approximate these curves by polynomials. This, however, proved to be practically impossible, since for various values of $M, E$, and $e$, the order and the coefficients of the polynomials would have to be selected differently. This, together with the fact that only one point is known analytically (i.e., $k_{E}^{e x p}$ ), leads to the selection of the piece-wise linear approximation (20).

\subsubsection{Verification}

To verify the empirical law (20), production lines (17) and (18) were simulated with parameters $M, E$, and $e$ other than those considered in Sections 4.1 and 4.2. Specifically, the following parameters have been selected: $M=5, E \in\{0.7,0.8,0.9\}$, $e \in\{0.6,0.7,0.8\}, T_{\text {down }}=10$. (In lines (18), the first 5 machines were selected.) The results are shown in Figure 11. As one can see, the upper bound given by (20) still holds.

\section{$5 L L B$ in serial lines with $C V_{u p} \neq C V_{\text {down }}$}

\subsection{Effect of $C V_{\text {up }}$ and $C V_{\text {down }}$}

The case of $C V_{u p} \neq C V_{\text {down }}$ is complicated by the fact that $C V_{u p}$ and $C V_{\text {down }}$ may have different effects on $k_{E}$. If this difference is significant, it would be difficult 
to expect that the empirical law (20) could be extended to the case of unequal coefficients of variation. On the other hand, if $C V_{u p}$ and $C V_{\text {down }}$ affect $k_{E}$ in a somewhat similar manner, it would seem likely that (20) might be extended to the case under consideration. Therefore, analysis of effects of $C V_{u p}$ and $C V_{\text {down }}$ on $k_{E}$ is of importance. This section is devoted to such an analysis.

To investigate this issue, introduce two functions:

$k_{E}\left(C V_{u p} \mid C V_{\text {down }}=\alpha\right)$

and

$k_{E}\left(C V_{\text {down }} \mid C V_{u p}=\alpha\right)$,

where

$\alpha \in\{0.1,0.25,0.5,0.75,1.0\}$.

Function (22) describes $k_{E}$ as a function of $C V_{u p}$ given that $C V_{d o w n}=\alpha$, while (23) describes $k_{E}$ as a function of $C V_{\text {down }}$ given that $C V_{u p}=\alpha$. If for all $\alpha$ and $\beta \in\{0.1,0.25,0.5,0.75,1.0\}$,

$k_{E}\left(C V_{\text {down }}=\beta \mid C V_{\text {up }}=\alpha\right)<k_{E}\left(C V_{\text {up }}=\beta \mid C V_{\text {down }}=\alpha\right)$

when $\alpha>\beta$, it must be concluded that $C V_{\text {down }}$ has a larger effect on $k_{E}$ than $C V_{u p}$. If the inequality is reversed, $C V_{u p}$ has a stronger effect. Finally, if (25) holds for some $\alpha$ and $\beta$ from (24) and does not hold for others, the conclusion would be that, in general, neither has a dominant effect.

To investigate which of these situations takes place, we evaluated functions (22) and (23) using the approach described in Section 3. Some of the results for Weibull distribution are shown in Figure 12 (where the broken lines and $C V_{\text {eff }}$ will be defined in Sect. 5.2). Similar results were obtained for gamma and log-normal distributions as well (see Enginarlar et al., 2003b for details). From these results, the following can be concluded:

- For all $\alpha$ and $\beta$, such that $\alpha>\beta$, inequality (25) takes place. Thus, $C V_{\text {down }}$ has a larger effect on $k_{E}$ than $C V_{u p}$.

- However, since each pair of curves (22), (23) corresponding to the same $\alpha$ are close to each other, the difference in the effects of $C V_{u p}$ and $C V_{d o w n}$ is not too dramatic. To analyze this difference, introduce the function

$$
\begin{aligned}
& \epsilon_{3}^{A}\left(C V \mid C V_{u p}=C V_{\text {down }}=\alpha\right) \\
& =\frac{k_{E}^{A}\left(C V_{u p}=C V \mid C V_{\text {down }}=\alpha\right)-k_{E}^{A}\left(C V_{\text {down }}=C V \mid C V_{u p}=\alpha\right)}{k_{E}^{A}\left(C V_{u p}=C V \mid C V_{\text {down }}=\alpha\right)} \cdot 100,
\end{aligned}
$$

where $A \in\{W, g, L N\}$. The behavior of this function for Weibull distribution is shown in Figure 13 (see Enginarlar et al., 2003b for gamma and log-normal distributions). Thus, the effects of $C V_{u p}$ and $C V_{\text {down }}$ on $k_{E}$ are not dramatically different (typically within $20 \%$ and no more than $40 \%$ ). 


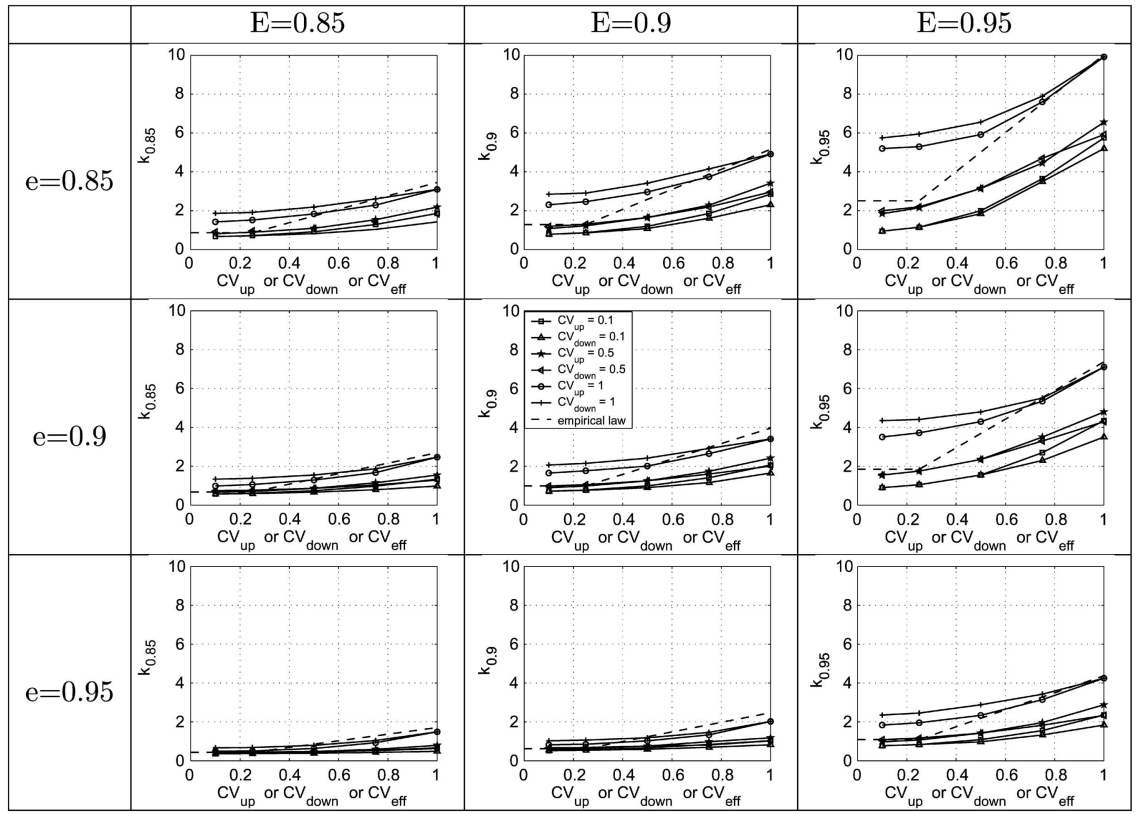

Fig. 12. $L L B$ versus $C V$ for $M=10$ Weibull machines

\subsection{Empirical law}

\subsubsection{Analytical expression}

Since the upper bound (20) is not too tight (and, hence, may accommodate additional uncertainties) and the effects of $C V_{u p}$ and $C V_{\text {down }}$ on $k_{E}$ are not dramatically different, the following extension of the empirical law is suggested:

$$
\begin{aligned}
& k_{E}\left(M, E, e, C V_{\text {up }}, C V_{\text {down }}\right) \\
& \leq \frac{\max \left\{0.25, C V_{u p}\right\}+\max \left\{0.25, C V_{\text {down }}\right\}}{2} k_{E}^{\exp }(M, E, e), \\
& C V_{\text {up }} \leq 1, \quad C V_{\text {down }} \leq 1,
\end{aligned}
$$

where, as before, $k_{E}^{e x p}$, is defined by (5), (6). If $C V_{u p}=C V_{d o w n},(27)$ reduces to (20); otherwise, it takes into account different values of $C V_{u p}$ and $C V_{\text {down }}$.

The first factor in the right-hand-side of (27) is denoted as $C V_{\text {eff }}$ :

$C V_{\text {eff }}=\frac{\max \left\{0.25, C V_{u p}\right\}+\max \left\{0.25, C V_{\text {down }}\right\}}{2}$.

Thus, (27) can be rewritten as

$k_{E} \leq C V_{e f f} k_{E}^{\exp }(M, E, e)$.

The right-hand-side of (29) is shown in Figure 12 by the broken lines.

The utilization of this law can be illustrated as follows: Suppose $C V_{u p}=0.1$ and $C V_{\text {down }}=1$. Then $C V_{\text {eff }}=0.625$ and, according to (27),

$k_{E} \leq 0.625 k_{E}^{\exp }(M, E, e)$. 


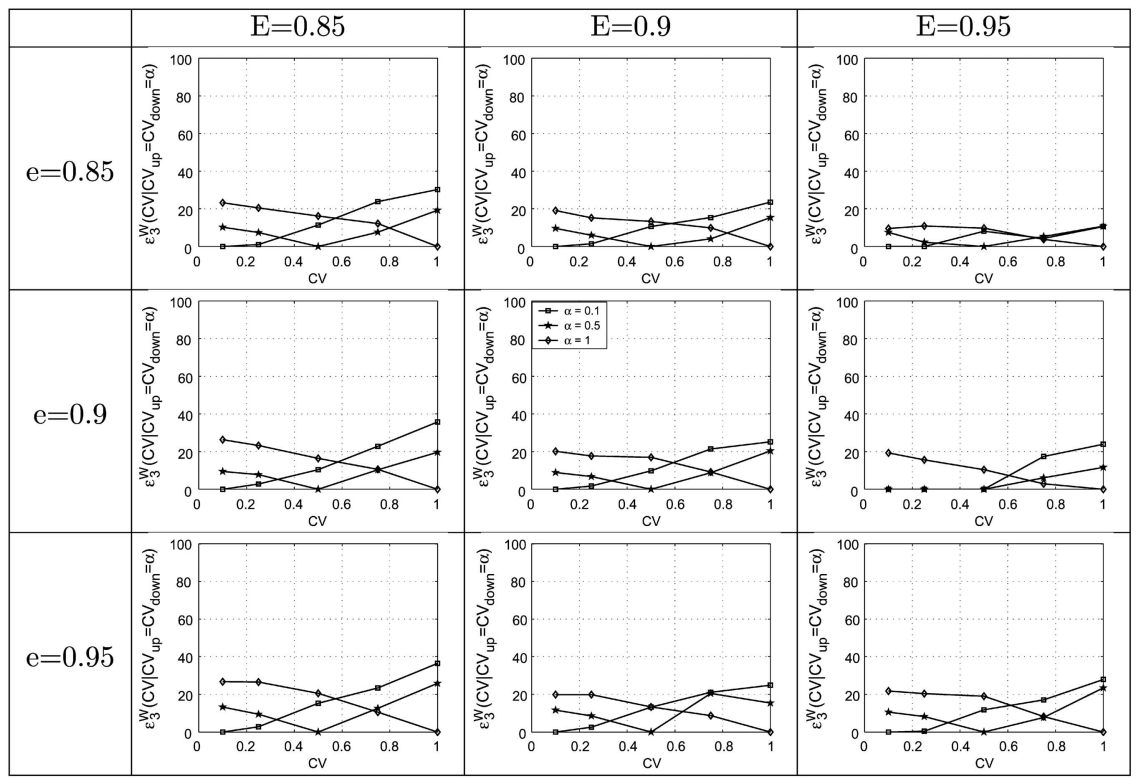

Fig. 13. Function $\epsilon_{3}^{W}\left(C V \mid C V_{u p}=C V_{\text {down }}=\alpha\right)$

Table 4. $\Delta(10, E, e)$ for all $C V_{u p} \neq C V_{\text {down }}$ cases considered

\begin{tabular}{cccc}
\hline & $E=0.85$ & $E=0.9$ & $E=0.95$ \\
\hline$e=0.85$ & 0.1016 & 0.0386 & 0.0687 \\
$e=0.9$ & 0.0425 & 0.1647 & 0.1625 \\
$e=0.95$ & 0.0402 & 0.0488 & 0.1200 \\
\hline
\end{tabular}

To investigate the validity of the empirical law (27), consider the following function:

$$
\begin{aligned}
\Delta(M, E, e)= & \min _{A \in\{(17)\}} \min _{C V_{u p}, C V_{\text {down }} \in\{(24)\}} \\
& {\left[k_{E}^{\text {upper bound }}\left(M, E, e, C V_{\text {eff }}\right)-k_{E}^{A}\left(M, E, e, C V_{u p}, C V_{\text {down }}\right)\right], }
\end{aligned}
$$

where $k_{E}^{\text {upper bound }}$ is the right-hand-side of (29), i.e.,

$k_{E}^{\text {upper bound }}\left(M, E, e, C V_{e f f}\right)=C V_{e f f} k_{E}^{e x p}(M, E, e)$.

If for all values of its arguments, function $\Delta(M, E, e)$ is positive, the right-handside of inequality (27) is an upper bound. The values of $\Delta(10, E, e)$ for $E \in$ $\{0.85,0.9,0.95\}$ and $e \in\{0.85,0.9,0.95\}$ are shown in Table 4. As one can see, function $\Delta(10, E, e)$ indeed takes positive values. Thus, the empirical law (27) takes place for all distributions and parameters analyzed. 


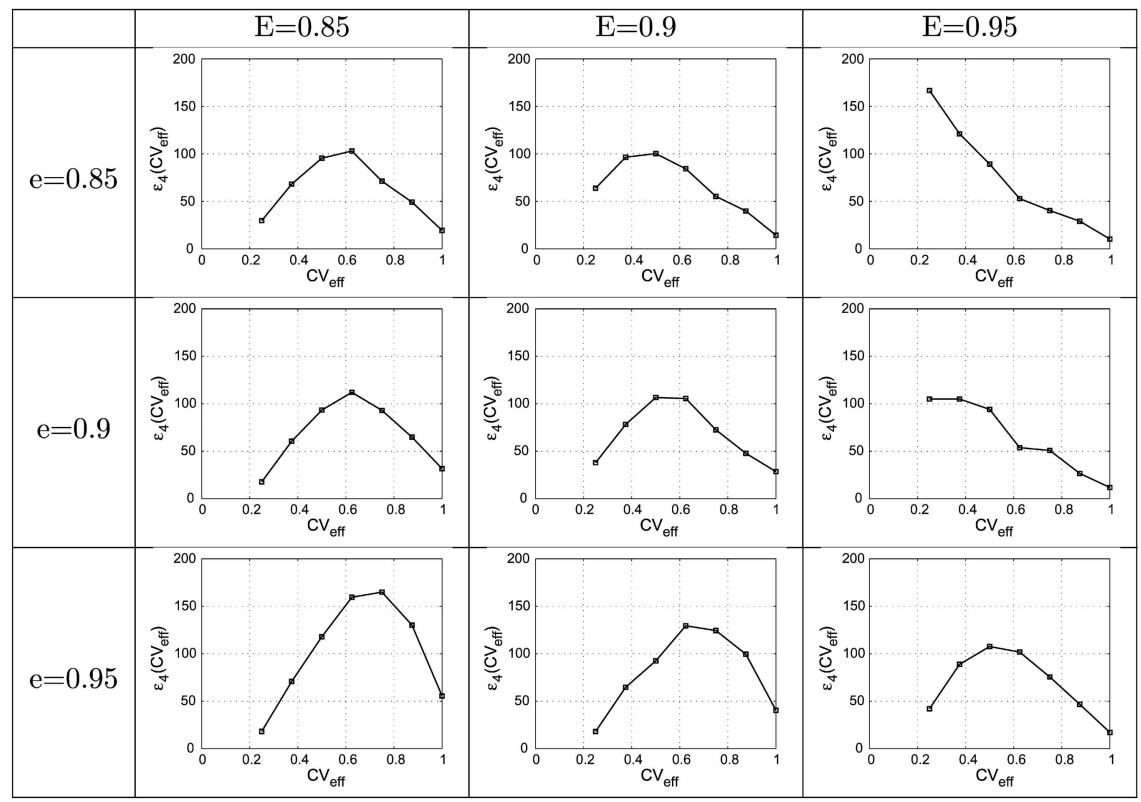

Fig. 14. The tightness of the empirical law (27)

To investigate the tightness of the bound (27), consider the function

$$
\begin{aligned}
\epsilon_{4}\left(C V_{e f f}\right)= & \max _{A \in\{(17)\} C V_{u p}, C V_{\text {down }} \in\{(24)\}} \\
& \frac{k_{E}^{\text {upperbound }}\left(M, E, e, C V_{\text {eff }}\right)-k_{E}^{A}\left(M, E, e, C V_{u p}, C V_{\text {down }}\right)}{k_{E}^{A}\left(M, E, e, C V_{u p}, C V_{\text {down }}\right)} \cdot 100 .
\end{aligned}
$$

Figure 14 illustrates the behavior of this function. Comparing this with Figure 10, we conclude that the tightness of bound (27) appears to be similar to that of (20).

\subsubsection{Verification}

To evaluate the validity of the upper bound (27), serial production lines with $M=5$, $E \in\{0.7,0.8,0.9\}, e \in\{0.6,0.7,0.8\}$, and $T_{u p}=10$ were simulated. For each of these parameters, systems (17) and (18) have been considered. (For system (18), the first 5 machines were selected.) Typical results are shown in Figure 15 (see Enginarlar et al., 2003b for more details). The validity of empirical law (27) for these cases is analyzed using function $\Delta(M, E, e)$, defined in (30) with the only difference that the first min is taken over $A \in\{(17),(18)\}$. Since the values of this function, shown in Table 5, are positive, we conclude that empirical law (27) is indeed verified for all values of $M, E, e$, and all distributions of up- and downtime considered. 


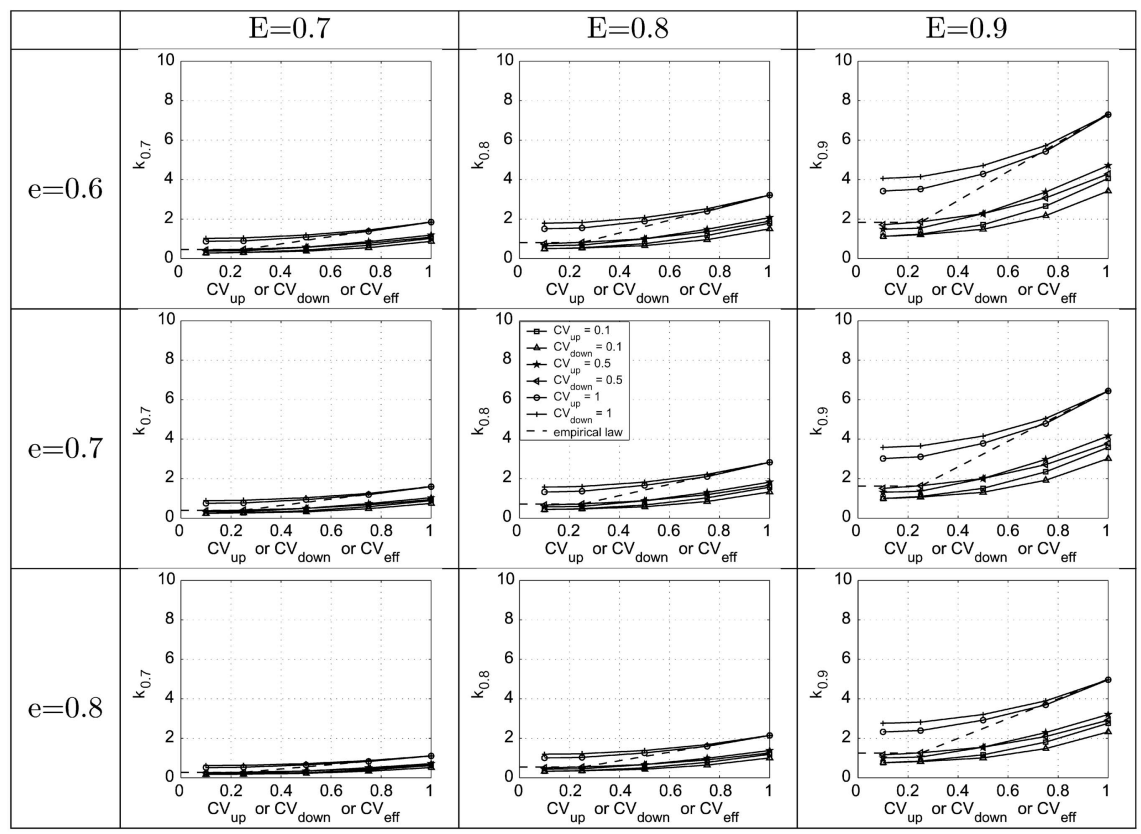

Fig. 15. Verification: $L L B$ versus $C V$ for $M=5$ Weibull machines

Table 5. Verification: $\Delta(5, E, e)$ for all $C V_{u p} \neq C V_{\text {down }}$ cases considered

\begin{tabular}{llll}
\hline & $E=0.7$ & $E=0.8$ & $E=0.9$ \\
\hline$e=0.6$ & 0.0039 & 0.0242 & 0.0547 \\
$e=0.7$ & 0.0102 & 0.0213 & 0.0481 \\
$e=0.8$ & 0.0084 & 0.0162 & 0.0355
\end{tabular}

\section{$6 \operatorname{SYSTEM}\left\{\left[G_{u p}, G_{\text {down }}\right]_{1}, \ldots,\left[G_{u p}, G_{\text {down }}\right]_{M}\right\}$}

So far, serial production lines with Weibull, gamma, and log-normal reliability models have been analyzed. It is of interests to extend this analysis to general probability density functions. Based on the results obtained above, the following conjecture is formulated:

The empirical laws (20) and (27) hold for serial production lines satisfying assumptions (i), (iii)-(vi) with up- and downtime having arbitrary unimodal probability density functions.

The verification of this conjecture is a topic for future research. 


\section{Conclusions}

Results described in this paper suggest the following procedure for designing lean buffering in serial production lines defined by assumptions (i)-(vi):

1. Identify the average value and the variance of the up- and downtime, $T_{u p}$, $T_{\text {down }}, \sigma_{u p}^{2}$, and $\sigma_{\text {down }}^{2}$, for all machines in the system (in units of machine cycle time). This may be accomplished by measuring the duration of the upand downtimes of each machine during a shift or a week of operation (depending on the frequency of occurrence). If the production line is at the design stage, this information may be obtained from the equipment manufacturer (however, typically with a lower level of certainty).

2. Using (5), (6), and $T_{\text {up }}, T_{\text {down }}$, determine the level of buffering, necessary and sufficient to obtain the desired efficiency, $E$, of the production line, if the downtime of all machines were distributed exponentially, i.e., $k_{E}^{e x p}$.

3. Finally, if $C V_{u p}=\frac{\sigma_{u p}}{T_{u p}} \leq 1$ and $C V_{\text {down }}=\frac{\sigma_{\text {down }}}{T_{\text {down }}} \leq 1$, evaluate the level of buffering for the line with machines under consideration using the empirical law

$k_{E} \leq \frac{\max \left\{0.25, C V_{u p}\right\}+\max \left\{0.25, C V_{\text {down }}\right\}}{2} \cdot k_{E}^{\text {exp }}$.

As it is shown in this paper, this procedure leads to a reduction of lean buffering by a factor of up to 4 , as compared with that based on the exponential assumption.

\section{References}

Altiok T (1985) Production lines with phase-type operation and repair times and finite buffers. International Journal of Production Research 23: 489-498

Altiok T (1989) Approximate analysis of queues. In: Series with phase-type service times and blocking. Operations Research 37: 601-610

Altiok T, Stidham SS (1983) The allocation of interstage buffer capacities in production lines. IIE Transactions 15: 292-299

Altiok T, Ranjan R (1989) Analysis of production lines with general service times and finite buffers: a two-node decomposition approach. Engineering Costs and Production Economics 17: 155-165

Buzacott JA (1967) Automatic transfer lines with buffer stocks. International Journal of Production Research 5: 183-200

Caramanis M (1987) Production line design: a discrete event dynamic system and generalized benders decomposition approach. International Journal of Production Research 25: 1223-1234

Chow W-M (1987) Buffer capacity analysis for sequential production lines with variable processing times. International Journal of Production Research 25: 1183-1196

Conway R, Maxwell W, McClain JO, Thomas LJ (1988) The role of work-in-process inventory in serial production lines. Operations Research 36: 229-241

Enginarlar E, Li J, Meerkov SM, Zhang RQ (2002) Buffer capacity to accommodating machine downtime in serial production lines. International Journal of Production Research 40: 601-624 
Enginarlar E, Li J, Meerkov SM (2003a) How lean can lean buffers be? Control Group Report CGR 03-10, Deptartment of EECS, University of Michigan, Ann Arbor, MI; accepted for publication in IIE Transactions on Design and Manufacturing (2005)

Enginarlar E, Li J, Meerkov SM (2003b) Lean buffering in serial production lines with non-exponential machines. Control Group Report CGR 03-13, Deptartment of EECS, University of Michigan, Ann Arbor, MI

Gershwin SB, Schor JE (2000) Efficient algorithms for buffer space allocation. Annals of Operations Research 93: 117-144

Harris JH, Powell SG (1999) An algorithm for optimal buffer placement in reliable serial lines. IIE Transactions 31: 287-302

Hillier FS, So KC (1991a) The effect of the coefficient of variation of operation times on the allocation of storage space in production line systems. IIE Transactions 23: 198-206

Hillier FS, So KC (1991b) The effect of machine breakdowns and internal storage on the performance of production line systems. International Journal of Production Research 29: 2043-2055

Inman RR (1999) Empirical evaluation of exponential and independence assumptions in queueing models of manufacturing systems. Production and Operation Management 8: 409-432

Jafari MA, Shanthikumar JG (1989) Determination of optimal buffer storage capacity and optimal allocation in multistage automatic transfer lines. IIE Transactions 21: 130-134

Li J, Meerkov SM (2005) On the coefficients of variation of up- and downtime in manufacturing equipment. Mathematical Problems in Engineering 2005: 1-6

Park T (1993) A two-phase heuristic algorithm for determining buffer sizes in production lines. International Journal of Production Research 31: 613-631

Powell SG (1994) Buffer allocation in unbalanced three-station lines. International Journal of Production Research 32: 2201-2217

Powell SG, Pyke DF (1998) Buffering unbalanced assembly systems. IIE Transactions 30: $55-65$

Seong D, Change SY, Hong Y (1995) Heuristic algorithm for buffer allocation in a production line with unreliable machines. International Journal of Production Research 33: 19892005

Smith JM, Daskalaki S (1988) Buffer space allocation in automated assembly lines. Operations Research 36: 343-357

Tempelmeier H (2003) Practical considerations in the optimization of flow production systems. International Journal of Production Research 41: 149-170

Yamashita H, Altiok T (1988) Buffer capacity allocation for a desired throughput of production lines. IIE Transactions 30: 883-891 